\title{
Implantação da Técnica de Criopreservação de Pré-embriões Humanos pelo Método Ultra-rápido
}

Autora: Iara Lima Couy

Orientador: Prof. Dr. Marcos Dias de Moura

Dissertação de Mestrado apresentada ao Departamento de Ginecologia e Obstetrícia na Faculdade de Medicina de Ribeirão Preto da Universidade de São Paulo, em 29 de maio de 2000.

No presente trabalho, avaliou-se a implantação da técnica de criopreservação de pré-embriões humanos pelo método ultra- rápido, no Setor de Reprodução Humana do Hospital das Clínicas da Faculdade de Medicina de Ribeirão Preto, Universidade de São Paulo. No período de setembro de 1997 a dezembro de 1999, 223 pacientes foram submetidas à transferência de pré-embriões (TE) a fresco, obtendo-se uma taxa de gestação por transferência de 40,3\% $(90 / 223)$. O congelamento de pré-embriões excedentes foi realizado em 50 pacientes, divididas em 2 grupos de acordo com o resultado da TE a fresco. O Grupo I $(n=26)$ correspondeu às pacientes que não engravidaram no ciclo de TE a fresco e o Grupo II $(n=24)$, às pacientes que engravidaram. Não houve diferença estatisticamente significativa com relação às características clínicas das pacientes e do ciclo de estimulação ovariana entre os dois grupos. A idade das pacientes, as causas de infertilidade, o número de oócitos captados, as taxas de fertilização e de clivagem e o número de pré-embriões transferidos a fresco não foram diferentes entre os dois grupos. O número e a qualidade morfológica dos pré-embriões congelados também não mostrou diferença entre os grupos. Durante o descongelamento, 94\% dos pré-embriões foram recuperados (95/101), sendo a taxa de sobrevivência de 50,5\% (48/95). Procedeu-se ao descongelamento de pré-embriões em 25 ciclos monitorizados, com transferência embrionária em 21 casos. Até o momento, não houve gestação. Os dados do presente trabalho sugerem que a técnica de congelamento e descongelamento embrionário pelo método ultra-rápido é exeqüivel e de baixo custo, apresentando resultados aceitáveis em um programa de Reprodução Assistida com relação à recuperação e sobrevivência embrionárias. Com o descongelamentos dos préembriões restantes poderemos avaliar, com um número maior de casos, o real benefício desta técnica no incremento das taxas de gestação por ciclo estimulado.

Palavras-chave: Fertilização in vitro. Criopreservação. Reprodução assistida.

\section{Comportamento da Necessidade de Insulina em Gestantes Diabéticas - Influência no Controle da} Glicemia Materna e no Resultado Perinatal.

Autor: Humberto Migiolaro

Orientador: Marilza Vieira Cunha Rudge

Dissertação de Mestrado apresentada ao curso de Pós-graduação em Ginecologia e Obstetrícia, Área de Concentração em Obstetrícia da Faculdade de Medicina de Botucatu, em 20 de novembro de 2000.

Justificativa: A insulino-resistência cresce progressivamente na gestação impondo ajustes periódicos da dose de insulina em grávidas diabéticas. O tipo de curva de insulinoterapia parece influenciar o resultado perinatal. Objetivo: Estudar a influência do comportamento da necessidade de insulina no controle da glicemia materna e no resultado perinatal.

Sujeitos e Métodos: Estudo retrospectivo das curvas de insulinoterapia de 193 gestantes diabéticas do grupo II-B (Rudge, 1983). De acordo com as curvas individuais, as variáveis independentes foram: necessidade de insulina crescente após 24-28 semanas (comportamento fisiológico), necessidade de insulina diminuindo, constante ou oscilatória após 24-28 semanas (comportamento não-fisiológico). As variáveis dependentes foram: média glicêmica da gestação, classificação dos recém-nascidos pelo peso e idade gestacional, Apgar-5 min, coeficiente de mortalidade perinatal e permanência hospitalar neonatal.

Resultados: O comportamento fisiológico ocorreu em
$79,3 \%$ das gestantes e resultou em queda progressiva das médias glicêmicas; com 25,7\% de recém-nascidos GIG, 49,3\% de prematuridade, média de 8,8 de Apgar$5 \mathrm{~min}, \mathrm{MPN}=59 / 1000$ e permanência hospitalar neonatal mais freqüente de 1 a 3 dias. No comportamento não-fisiológico a média glicêmica caiu após 28 semanas, mantendo-se estável até o final; ocorreram $42,8 \%$ de recém-nascidos GIG, $67,9 \%$ de prematuridade, média 7,0 de Apgar-5 min, MPN=300/1000 e permanência hospitalar neonatal mais freqüente maior de 7 dias.

Conclusões: 1) O comportamento fisiológico da necessidade de insulina foi mais freqüente, associou-se com queda progressiva da média glicêmica materna e melhor resultado perinatal. 2) O comportamento não-fisiológico associou-se com pior controle glicêmico materno e pior resultado perinatal.

Palavras-chave: Diabete melito. Prematuridade. Mortalidade perinatal. 


\title{
Transporte Eritrocitário de L-Arginina na Gestação e Período Pós-Parto
}

Autora: Adriani Oliveira Galão

Orientadora: Bartira E. Pinheiro Da Costa

Dissertação de Mestrado apresentada ao Curso de Pós-graduação em Clínica Médica, Área de Concentração: Nefrologia na Universidade Católica do Rio Grande do Sul em 01 março de 2000.

O óxido nítrico é um potente vasodilatador, sintetizado a partir do aminoácido L-arginina. Há evidências de alterações na via L-arginina-óxido nítrico na gestação, mas sua importância na gênese da pré-eclâmpsia é desconhecida. Pouco se sabe sobre o transporte do aminoácido pela membrana celular ao longo da gestação. A capacidade máxima de transporte de L-arginina parece estar aumentada na pré-eclâmpsia superposta à hipertensão, mas não na pré-eclâmpsia.

O objetivo deste estudo foi avaliar o transporte de Larginina na gravidez e no período pós-parto em uma coorte. A amostra foi composta por cento e oito gestantes hígidas, acompanhadas no segundo e terceiro trimestres e pós-parto, separadas em três grupos: primigestas, multíparas e pré-eclâmpticas. O influxo eritrocitário foi medido com L-arginina marcada com ${ }^{14} \mathrm{C}$ (a $37^{\circ} \mathrm{C}$, por 3 minutos). Resultados submetidos à equação de Michaelis-Menten, para determinar a ca- pacidade máxima de transporte $\left(\mathrm{V}_{\max }\right)$ e a constante de meia saturação $\left(\mathrm{K}_{\mathrm{m}}\right)$. ANOVA, complementado por Tukey, foi empregado na análise estatística; para avaliar associações de $\mathrm{V}_{\max }$ e $\mathrm{K}_{\mathrm{m}}$ com outras variáveis, utilizou-se o coeficiente de correlação de Pearson. O nível de significância adotado foi $\alpha=0,05$. A incidência de pré-eclâmpsia foi de 4,6\% (IC95\%: 1,5 a 10,5\%). $\mathrm{V}_{\max }$ aumentou significativamente durante as gestações $\mathrm{e}$ pós-parto, em gestantes normais. Na pré-eclâmpsia, ocorreu diferença apenas do segundo trimestre para os outros períodos. $\mathrm{K}_{\mathrm{m}}$ aumentou no pós-parto nos três grupos, e também ao longo da gestação nas multíparas normais. $\mathrm{V}_{\max }$ e $\mathrm{K}_{\mathrm{m}}$ foram similares nos três grupos. Não houve correlação entre parâmetros cinéticos e evolução clínica, laboratorial e puerperal.

Palavras-chave: Óxido Nítrico. Hipertensão arterial. L-arginina.

\section{Estudo da Variação da Inibina B como Fator Prognóstico da Intensidade da Resposta Ovariana à Hiperestimulação Controlada nos Programas de Fertilização in vitro.}

\author{
Autor: Artur Dzik \\ Orientador: Prof. Dr. Vicente Mario Izzo
}

Tese de Doutorado apresentada ao Departamento de Ginecologia e Obstetrícia da FMUSP, em 17 de outubro de 2000.

A técnica de fertilização in vitro e transferência de embriões na terapêutica de casais inférteis surgiu há 22 anos e hoje contabiliza mais de 300.000 nascimentos em 50 países diferentes. Neste trabalho descrevemos um novo teste de estímulo para avaliação da reserva ovariana, através da estimulação ovariana pontual com 300 UI de FSHp no 3ํ dia do ciclo menstrual, avaliando a intensidade da resposta ovariana pelo D inibina B / $24 \mathrm{~h}$ no screening de pacientes boas respondedoras e más respondedoras em 32 ciclos terapêuticos de FIVeTE. As pacientes foram submetidas à hiperestimulação ovariana controlada com gonadotrofina da mulher menopausada sob bloqueio do eixo hipotálamohipófise-ovário prévio com análogo agonista do hormônio liberador das gonadotrofinas. A média da faixa etária foi de 34,0 $\pm 3,7$ anos. A indicação da FIVeTE foi fator tubo-peritoneal $(76,0 \%)$, fator masculino $(18,0 \%)$ e fator idiopático (6,0\%). As variáveis FSH, $\Delta$ estradiol/ $24 \mathrm{~h}$, inibina $\mathrm{B}$ basal e $\Delta$ Inibina $\mathrm{B} / 24 \mathrm{~h}$ apresentaram nivel descritivo de 0,0256, 0,0215, 0,0142 e 0,0053 respectivamente no screening das pacientes boas respondedoras e más respondedoras. Nossos resultados sugerem que a avaliação do $\Delta$ inibina $\mathrm{B} / 24 \mathrm{~h}$ em resposta ao teste de estimulo ovariano com FSHp foi estatisticamente superior à dosagem do FSH basal, $\Delta$ estradiol/24 h e inibina B basal sendo, portanto, mais adequado no screening de pacientes boas respondedoras e más respondedoras.

Palavras-chave: Inibina B. Reserva Ovariana. Infertilidade. Fertilização in vitro. 